\title{
A multi-laboratory comparison of photon migration instruments and their performances: the BitMap exercise
}

\section{Lanka, Sri Rama Pranav Kumar, Yang, Lin, Orive-Miguel, David, Deepak Veesa, Joshua, Tagliabue, Susanna, et al.}

Sri Rama Pranav Kumar Lanka, Lin Yang, David Orive-Miguel, Joshua Deepak Veesa, Susanna Tagliabue, Aleh Sudakou, Saeed Samaei, Mario Forcione, Zuzana Kovacsova, Anurag Behera, Thomas Gladytz, Dirk Grosenick, Lionel Hervé, Giuseppe Lo Presti, Lorenzo Cortese, Turgut Durduran, Karolina Bejm, Magdalena Morawiec, Piotr Sawosz, Michal Kacprzak, Anna Gerega, Adam Liebert, Antonio Belli, Ilias Tachtsidis, Frédéric Lange, Gemma Bale, Luca Baratelli, Sylvain Gioux, Alexander Kalyanov, Martin Wolf, Sanathana Konugolu Venkata Sekar, Marta Zanoletti, Ileana Pirovano, Michele Lacerenza, Lina Qiu, Edoardo Ferocino, Giulia Maffeis, Caterina Amendola, Lorenzo Colombo, Mauro Buttafava, Marco Renna, Laura Di Sieno, Rebecca Re, Andrea Farina, Lorenzo Spinelli, Alberto Dalla Mora, Davide Contini, Alessandro Torricelli, Alberto Tosi, Paola Taroni, Hamid Dehghani, Heidrun Wabnitz, Antonio Pifferi, "A multi-laboratory comparison of photon migration instruments and their performances: the BitMap exercise," Proc. SPIE 11639, Optical Tomography and Spectroscopy of Tissue XIV, 116390F (5 March 2021); doi: 10.1117/12.2578521 


\title{
A multi-laboratory comparison of photon migration instruments and their performances - the BitMap Exercise
}

\author{
Pranav Lanka ${ }^{\mathrm{a}}$, Lin Yang ${ }^{\mathrm{b}}$, David Orive-Miguel ${ }^{\mathrm{c}}$, Joshua Deepak Veesa ${ }^{\mathrm{d}}$, Susanna Tagliabue ${ }^{\mathrm{e}}$, Aleh Sudakou ${ }^{\mathrm{f}}$, \\ Saeed Samaei ${ }^{\mathrm{f}}$, Mario Forcione ${ }^{\mathrm{g}}$, Zuzana Kovacsova ${ }^{\mathrm{h}}$, Anurag Behera ${ }^{\mathrm{a}}$, Thomas Gladytz ${ }^{\mathrm{b}}$, Dirk Grosenick ${ }^{\mathrm{b}}$, \\ Lionel Hervéc, Giuseppe LoPresti ${ }^{\mathrm{e}}$, Lorenzo Cortese ${ }^{\mathrm{e}}$, Turgut Durduran ${ }^{\mathrm{e}}$, Karolina Bejm ${ }^{\mathrm{f}}$, Magdalena \\ Morawiec $^{\mathrm{f}}$, Michał Kacprzak ${ }^{\mathrm{f}}$, Piotr Sawosz ${ }^{\mathrm{f}}$, Anna Grega ${ }^{\mathrm{f}}$, Adam Liebert ${ }^{\mathrm{f}}$, Antonio Belli ${ }^{\mathrm{g}}$, Ilias Tachtsidis ${ }^{\mathrm{h}}$, \\ Frederic Lange ${ }^{\mathrm{h}}$, Gemma Bale ${ }^{\mathrm{h}}$, Luca Baratelli ${ }^{\mathrm{i}}$, Sylvain Gioux ${ }^{\mathrm{i}}$, Kalyanov Alexander ${ }^{\mathrm{j}}$, Martin Wolf $^{\mathrm{j}}$, , \\ Sanathana Konugolu Venkata Sekark ${ }^{\mathrm{k}}$, Marta Zanolettia ${ }^{\mathrm{a}}$ Ileana Pirovano ${ }^{\mathrm{a}}$, Michele Lacerenza ${ }^{\mathrm{a}}$, Lina Qiu $^{\mathrm{a}}$, \\ Edoardo Ferocino $^{a}$, Giulia Maffeis ${ }^{\mathrm{a}}$, Caterina Amendola ${ }^{\mathrm{a}}$, Lorenzo Colombo ${ }^{\mathrm{a}}$, Mauro Buttafava ${ }^{\mathrm{m}}$, Marco \\ Renna $^{\mathrm{m}}$, Laura Di Sieno ${ }^{\mathrm{a}}$, Rebecca Re ${ }^{\mathrm{a}}$, Andrea Farina ${ }^{1}$, Lorenzo Spinelli ${ }^{1}$, Alberto Dalla Mora ${ }^{\mathrm{a}}$, Davide \\ Contini $^{\mathrm{a}}$, Paola Taroni ${ }^{\mathrm{a}}$, Alberto Tosi ${ }^{\mathrm{m}}$, Alessandro Torricelli ${ }^{\mathrm{a}}$, Hamid Dehghani ${ }^{\mathrm{d}}$, Heidrun Wabnitz ${ }^{\mathrm{b}}$, and \\ Antonio Pifferi ${ }^{\mathrm{a}}$ \\ ${ }^{\text {aP }}$ olitecnico di Milano, Dipartimento di Fisica, Milano, Italy. \\ ${ }^{b}$ Physikalisch-Technische Bundesanstalt (PTB), Berlin, Germany. \\ ${ }^{c}$ CEA-Leti: Laboratoire d'électronique des technologies de l'information, Grenoble, France. \\ ${ }^{\mathrm{d}}$ School of Computer Science, University of Birmingham, Birmingham, UK. \\ ${ }^{\mathrm{e} T}$ The Institute of Photonic Sciences(ICFO), Castelldefels, Spain. \\ ${ }_{\mathrm{f}}$ Nalecz Institute of Biocybernetics and Biomedical Engineering, Warsaw, Poland. \\ gNational Institute for Health Research Surgical Reconstruction and Microbiology Research Centre, \\ University Hospitals Birmingham, UK. \\ ${ }^{\mathrm{h}}$ Medical Physics \& Biomedical Engineering, UCL, London, UK \\ ${ }^{i}$ University of Strasbourg, Strasbourg, France \\ ${ }^{\mathrm{j} B i o m e d i c a l ~ O p t i c s ~ R e s e a r c h ~ L a b o r a t o r y, ~ D e p a r t m e n t ~ o f ~ N e o n a t o l o g y, ~ U n i v e r s i t y ~ H o s p i t a l ~ Z u r i c h, ~}$ \\ Zurich, Switzerland \\ kIPIC, Tyndall National Institute, Cork, Ireland; \\ ${ }^{1}$ Istituto di Fotonica e Nanotecnologie, Milano, Italy \\ mipartimento di Elettronica, Informazione e Bioingegneria, Politecnico di Milano, Italy
}

\begin{abstract}
Performance assessment and standardization are indispensable for instruments of clinical relevance in general and clinical instrumentation based on photon migration/diffuse optics in particular. In this direction, a multi-laboratory exercise was initiated with the aim of assessing and comparing their performances. 29 diffuse optical instruments belonging to 11 partner institutions of a European level Marie Curie Consortium BitMap ${ }^{1}$ were considered for this exercise. The enrolled instruments covered different approaches (continuous wave, $\mathrm{CW}$; frequency domain, $\mathrm{FD}$; time domain, TD and spatial frequency domain imaging, SFDI) and applications (e.g. mammography, oximetry, functional imaging, tissue spectroscopy). 10 different tests from 3 well-accepted protocols, namely, the MEDPHOT ${ }^{2}$, the $\mathrm{BIP}^{3}$, and the nEUROPt ${ }^{4}$ protocols were chosen for the exercise and the necessary phantoms kits were circulated across labs and institutions enrolled in the study. A brief outline of the methodology of the exercise is presented here. Mainly, the design of some of the synthetic descriptors, (single numeric values used to summarize the result of a test and facilitate comparison between instruments) for some of the tests will be discussed.. Future actions of the exercise aim at deploying these measurements onto an open data repository and investigating common analysis tools for the whole dataset.
\end{abstract}

Keywords: performance assessment, standardization, diffuse optics, near-infrared, phantom, absorption, scattering

Optical Tomography and Spectroscopy of Tissue XIV, edited by Sergio Fantini, Paola Taroni, Proc. of SPIE Vol. 11639, 116390F - (c) 2021 SPIE · CCC code: 1605-7422/21/ $\$ 21 \cdot$ doi: 10.1117/12.2578521 


\section{INTRODUCTION}

Near-infrared spectroscopy (also known as diffuse optical spectroscopy) is a technique that uses the physics of photon migration within tissue to monitor and image different parts of the human body in-vivo. Its low-cost, portable, noninvasive, and real-time nature enabled the development of a wide variety of diffuse optics-based instrumentation for varied purposes. However, a major bottleneck in the path towards commercialization of this technology is the pressing need for performance assessment and standardization. Having robust and standardized performance assessment methodologies help in the quality assurance of the instrument at a clinical level and allow for easy re-calibration if necessary.

The BitMap exercise is an initiative aimed at enforcing such a culture of performance assessment at a multi-laboratory level. A total of 29 instruments, from 11 institutions spread across 7 EU countries were enrolled for this exercise. 10 different tests from 3 well-accepted protocols, namely, the MEDPHOT ${ }^{2}$, the BIP ${ }^{3}$, and the $\mathrm{nEUROPt}^{4}$ protocols were chosen for the exercise and the necessary phantoms kits (see Fig.1) were circulated across labs and institutions enrolled in the study. A classification of the instruments based on the modality and application is presented in Table 1 . The exercise is broken down into three actions aimed at

1) Measure and compare individual instrument performance against the phantoms and protocols.

2) Deployment of the data into an open data repository

3) Testing the resulting dataset against standard analysis models.

Table 1. Overview of the instruments enrolled for the exercise.

\begin{tabular}{|c|c|c|c|c|c|c|}
\hline \multirow{2}{*}{ Application } & \multicolumn{4}{|c|}{ Modality } & \multirow{2}{*}{ Total } \\
\cline { 2 - 7 } & CW & TD & FD & SFDI & \\
\hline Spectroscopy & 1 & 9 & 1 & 0 & 11 \\
\hline Imaging & 0 & 4 & 0 & 1 & 5 \\
\hline Oximetry & 2 & 8 & 1 & 0 & 11 \\
\hline DCS & 0 & 2 & 0 & 0 & 2 \\
\hline Total & 3 & 23 & 2 & 1 & $\mathbf{2 9}$ \\
\hline
\end{tabular}

DCS = Diffuse Correlation Spectroscopy, CW = Continuous Wave, TD = Time Domain, FD = Frequency Domain, SFDI = Spatial Frequency Domain Imaging

Action 1 of the BitMap exercise, particularly the MEDPHOT protocol (which involves 32 phantoms), results in a substantial amount of data for each instrument for each test. This prohibits an easy yet quantitative comparison of the results of the test between the different instruments considered in the exercise. Thus, there is a need for a single numeric value that could effectively summarize the result of a given test. This entity, known henceforth as the synthetic descriptor/ synthetic indicator, can then be used to compare the performance of the different instruments against that particular test. In this work, we discuss in detail the design and comparison of the synthetic descriptor for the Linearity and Coupling test of the MEDPHOT protocol. This is particularly important as this test alone invokes the use of all the 32 phantoms of the MEDPHOT kit.

\section{RESULTS}

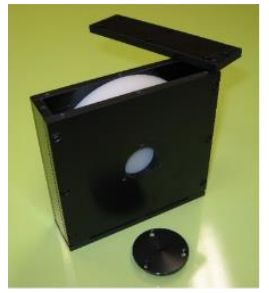

(a)

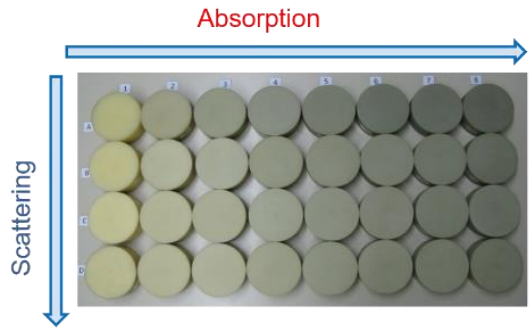

(b)

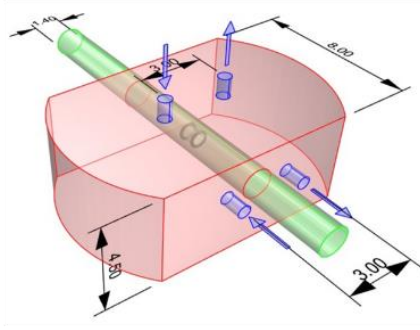

(c)

Figure 1. The (a) Responsivity phantom used in the BIP protocol. The (b) entire MEDPHOT kit comprising of 4 scattering (rows) and 8 absorption (columns) series and the (c) solid switchable phantom used in the nEUROPt protocol for the contrast measurements. 
A detailed description of the three protocols and an overview of the global methodology of the BitMap exercise is available here $^{2-5}$. In this work, we will particularly deal with the Linearity and Coupling assay of the MEDPHOT protocol.

The Linearity test is important to check whether the system can follow changes in a given parameter $\left(\mu_{a}\right.$ or $\left.\mu_{s}^{\prime}\right)$ without distortions. It is particularly important in spectroscopy, where preserving the shape of the spectrum is essential to accurately estimate the relative abundance of tissue constituents. Coupling, on the other hand, tests for the extent of absorption-toscattering coupling and vice versa, which can produce artifacts and cause deformations of the optical property spectra.

The MEDPHOT kit (Figure 1 (b)) comprises 32 phantoms labeled with a letter and a number. The letter stands for the nominal scattering (A, B, C, D corresponding to $\mu_{s}^{\prime}$ to roughly $5,10,15,20 \mathrm{~cm}^{-1}$ respectively @ $800 \mathrm{~nm}$ ) and the number indicates the absorption $\left(1,2,3,4,5,6,7,8\right.$ correspond to $\mu_{a}$ of $0,0.05,0.10,0.15,0.20,0.25,0.30,0.35 \mathrm{~cm}^{-1}$ respectively). Measuring all the phantoms (at a single wavelength) and plotting them as shown in Figure 2 (and Figure 3) we obtain the result for the Linearity and Coupling tests of the MEDPHOT protocol. While a wealth of information regarding a particular instrument can be deduced by these plots, it is quite challenging to use this to compare the results between different instruments.

The synthetic descriptors used to generate a comparative figure of merit plot for Linearity and Coupling in the BitMap exercise (Figure 4) presented in the title of each of the subplots of Figure 2 and Figure 3 are obtained as follows.

Linearity ( $\mathrm{a}, \mathrm{b}$ in Figures $2 \& 3$ ): The median value of the relative deviation between the data-points (filled circles) and the linear fit (dashed line) over the different series is considered to represent the median deviation from linearity for the specific optical property.

Coupling (c,d in Figures 2\&3): The median value of the absolute slopes of the linear fit (dashed line) over the different series is considered to represent the coupling between the two optical properties.

As the corresponding titles suggest, the deviation in linearity is given as a percentage value while the coupling is presented in $\mathrm{cm}^{-1}$ as a relative change in the measured optical property corresponding to a fixed variation in the other optical property $\left(0.01 \mathrm{~cm}^{-1}\right.$ of $\mu_{a}$ and $1 \mathrm{~cm}^{-1}$ of $\left.\mu_{s}^{\prime}\right)$.
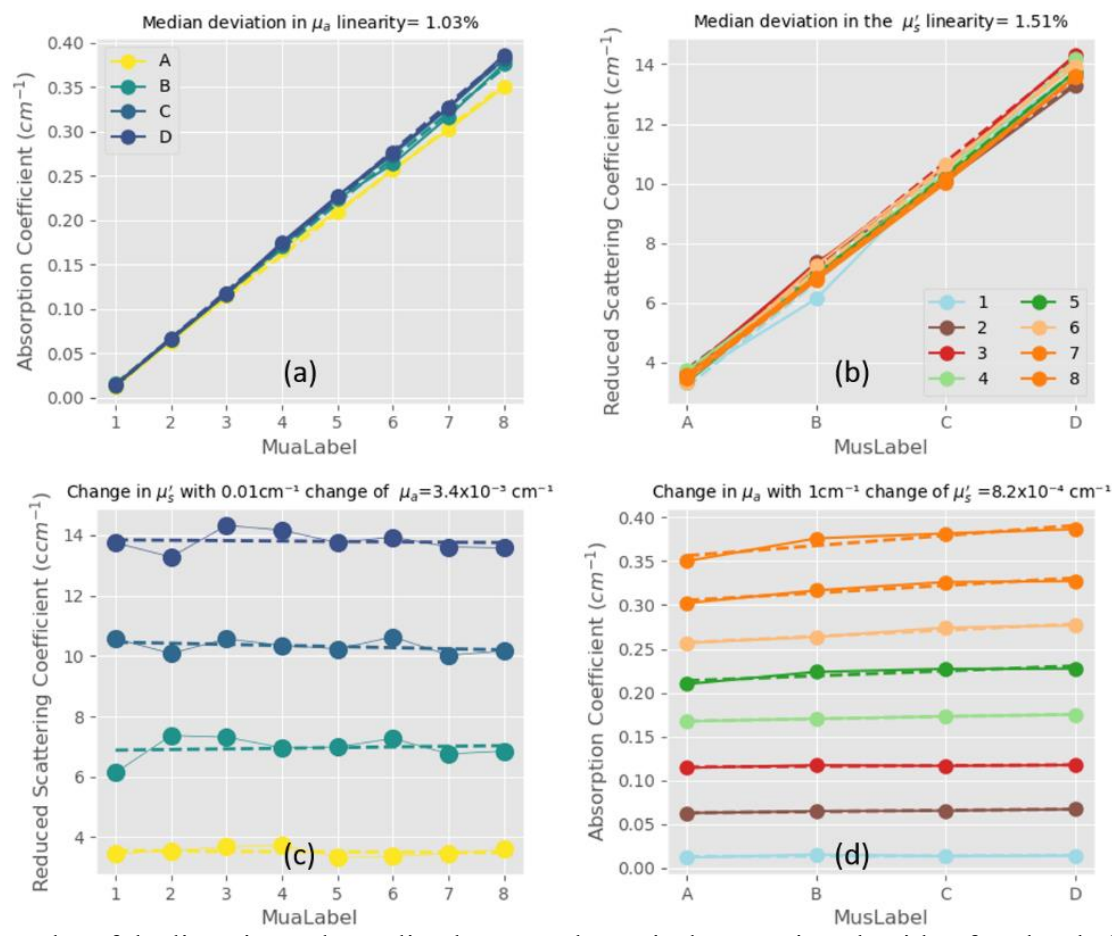

Figure 2. An exemplary plot of the linearity and coupling between the optical properties. The title of each subplot describes the synthetic indicator of the linearity or coupling observed which will be used for the figure of merit plot (Figure 4). 
According to these definitions, the ideal instrument would have four of these values as close to zero as possible. Two examples cases from the BitMap exercise, one which fares well in both the tests and one which relatively underperforms in both the tests are presented in Figures 2 and 3 respectively.
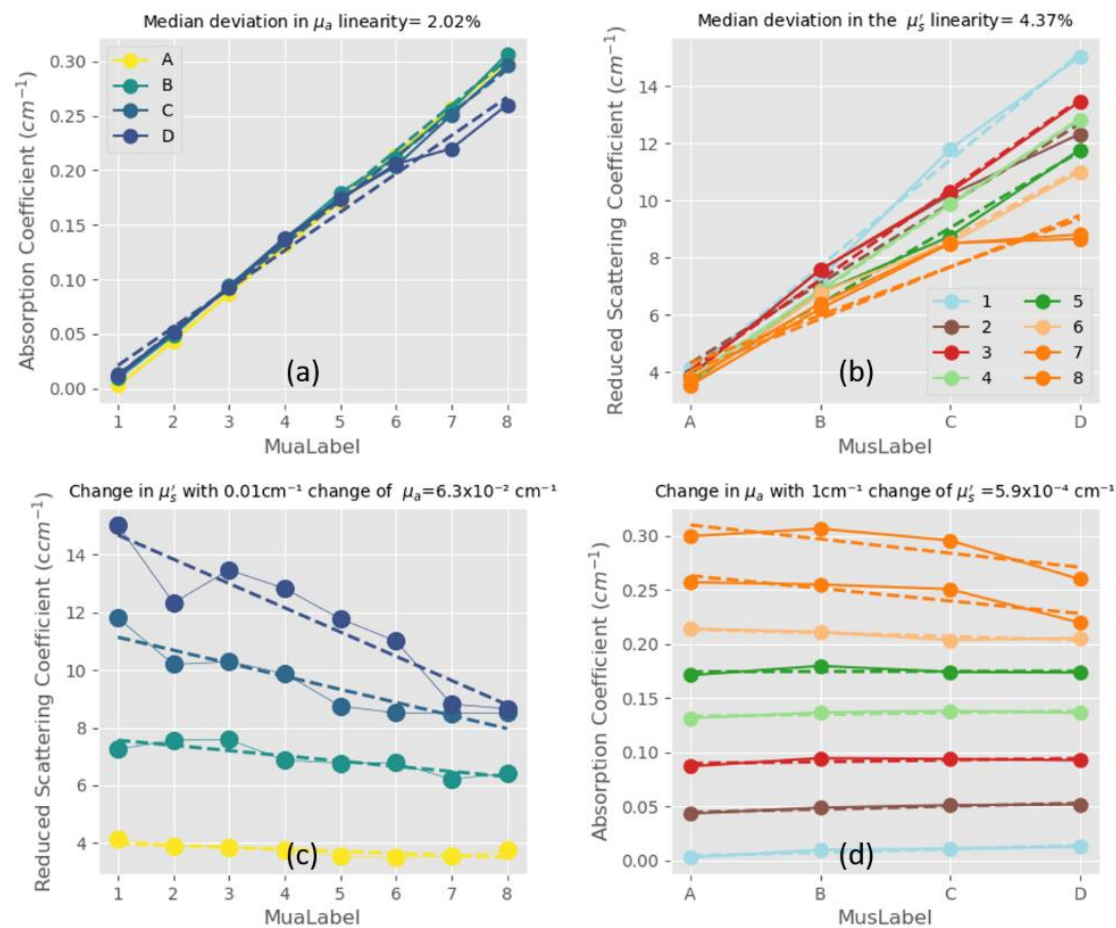

Figure 3. A second exemplary plot of the linearity and coupling between the optical properties.
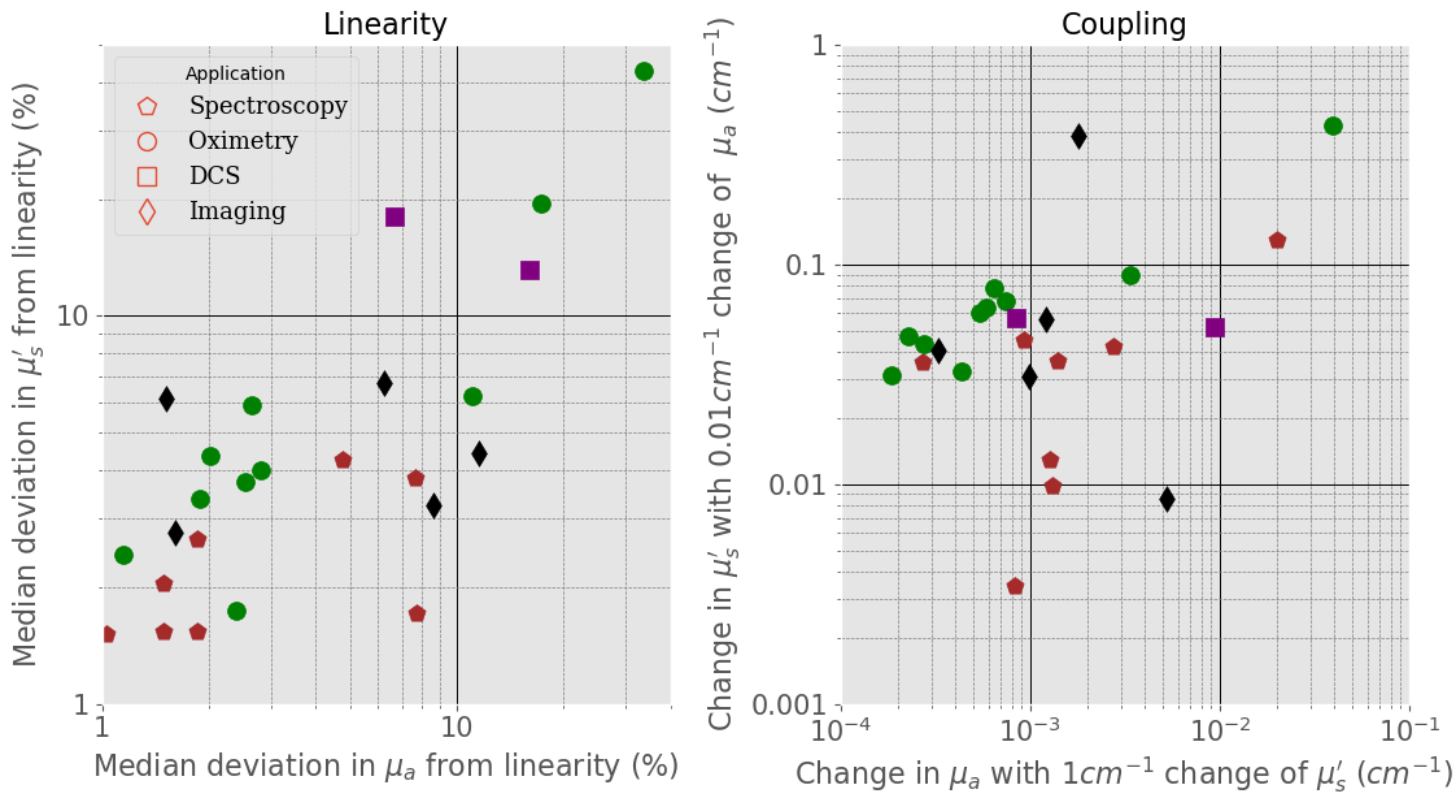

Figure 4. Figure of Merit plots for the (left) Linearity and (right) Coupling tests of the MEDPHOT protocol. The $\mathrm{x}$ and $\mathrm{y}$ axes in both cases are plotted on log scales. 
Not all the 29 instruments enrolled for the exercise are eligible for all the tests employed in the exercise. The design and physics behind some of the instruments disqualify them from certain tests. Each data point in Figure 4 is representative of the response of one instrument enrolled in the exercise. The spread of the data points in both the subplots suggests that the deviation in the linearity of both the optical properties for most of the instruments (> 85\%) is under $10 \%$. Similarly, most instrumentation experience a variation of at most $0.1 \mathrm{~cm}^{-1}$ in $\mu_{s}^{\prime}$ with a $0.01 \mathrm{~cm}^{-1}$ change in $\mu_{a}$ and within $0.05 \mathrm{~cm}^{-1}$ variation in $\mu_{a}$ with $1 \mathrm{~cm}^{-1}$ in $\mu_{s}^{\prime}$. Another interesting thing to note here would be the fact that most of the pentagons (which corresponding to the spectroscopy application) are close to the bottom left corners of both the subplots (the regions for the ideal performance). This hints at the fact that spectrometers by design outperform other kinds of diffuse optics instrumentation in this test.

\section{CONCLUSIONS}

In conclusion, 29 diffuse optics instruments from 11 different institutions across $7 \mathrm{EU}$ nations were enrolled in a largescale multi-laboratory performance assessment exercise. A total of 10 tests based on 3 international protocols were employed and the preliminary data analysis was performed. The methodology involved in the design of a comparable metric for one of the 10 tests, namely the Linearity and Coupling assay of the MEDPHOT protocol was presented in detail. Future actions of the exercise aim at deploying these measurements onto an open data repository and investigating common analysis tools for the whole dataset.

\section{ACKNOWLEDGEMENTS}

This work was supported by the European Union's Horizon 2020 Marie Sklodowska-Curie Innovative Training Networks (ITN-ETN) program, under grant agreement no 675332 BitMap.

\section{REFERENCES}

[1] "BitMap ITN - Home," < http://www.bitmap-itn.eu/> (20 January 2019).

[2] Pifferi, A., Torricelli, A., Bassi, A., Taroni, P., Cubeddu, R., Wabnitz, H., Grosenick, D., Möller, M., Macdonald, R., et al., "Performance assessment of photon migration instruments: the MEDPHOT protocol," Applied Optics 44(11), 2104 (2005).

[3] Wabnitz, H., Taubert, D.R., Mazurenka, M., Steinkellner, O., Jelzow, A., Macdonald, R., Milej, D., Sawosz, P., Kacprzak, M., et al., "Performance assessment of time-domain optical brain imagers, part 1: basic instrumental performance protocol,” Journal of Biomedical Optics 19(8), 086010 (2014).

[4] Wabnitz, H., Jelzow, A., Mazurenka, M., Steinkellner, O., Macdonald, R., Milej, D., Zolek, N., Kacprzak, M., Sawosz, P., et al., "Performance assessment of time-domain optical brain imagers, part 2: nEUROPt protocol," Journal of Biomedical Optics 19(8), 086012 (2014).

[5] Lanka, P., Yang, L., Orive-Miguel, D., Veesa, J.D., Tagliabue, S., Sudakou, A., Samaei, S., Forcione, M., Kovacsova, Z., et al., "The BITMAP exercise: a multi-laboratory performance assessment campaign of diffuse optical instrumentation," in Diffus. Opt. Spectrosc. Imaging VII 11074, H. Dehghani and H. Wabnitz, Eds., 44 (2019). 